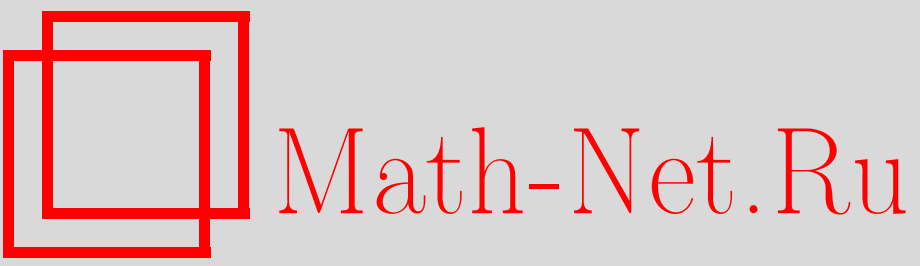

С. В. Лимаренко, Расширенная теорема плотности для градуированных по группе колец и модулей, УМH, 2002, том 57, выпуск 4, 181-182

DOI: https://doi.org/10.4213/rm544

Использование Общероссийского математического портала Math-Net.Ru подразумевает, что вы прочитали и согласны с пользовательским соглашением

http://www . mathnet.ru/rus/agreement

Параметры загрузки:

IP: 3.81 .55 .215

26 апреля 2023 г., 13:50:48 


\title{
РАСШИРЕННАЯ ТЕОРЕМА ПЛОТНОСТИ ДЛЯ ГРАДУИРОВАННЫХ ПО ГРУППЕ КОЛЕЦ И МОДУЛЕЙ
}

\author{
С.В. ЛИМАРЕНКО
}

Главным объектом рассмотрения в данной работе является расширенная теорема плотности для градуированных колец и модулей (теорема для неградуированного случая была опубликована в работе [1]), сфформулированная в терминах "ровной" однородности, и те дополнительные условия на градуированные кольцо и модуль над ним, при которых все три условия в формулировке теоремш становятся равносильными. В работе [2] доказывается расширенная теорема плотности, в которой условия приводятся в несколько иной форме, а градуировка производится по полугруппе. Мы же будем считать, что градуировка кольца и модуля производится по одной и той же аддитивной абелевой группе. В этом случае общность изложения мы приносим в жертву ясности и акцентированности основного результата. Чтобы избежать путаницы с индексами нумерации, мы обозначаем индексы однородности (элементы группы) строчными буквами греческого алфавита. Кольцо целых чисел обозначается $\mathbb{Z}$.

Градуированньй модуль назьвается сжимаемым, если он однородно вкладьвается в любой свой ненулевой градуированный подмодуль. Однородное вложение осуществляется однородньм мономорфизмом. Сжимаемьй модуль назьвается критически сжимаемьм, если он не допускает однородного вложения ни в какой свой градуированньй фактор-модуль. Свойства инъективности и квази-инъективности градуированных модулей будем формулировать в терминах однородных гомоморфизмов.

Следует заметить, что при однородном вложении градуированного модуля $M_{R}$ в $N_{R}$ (кольцо и градуировка одни и те же у обоих модулей) однородные компоненты могут лишь сдвигаться на элемент группы, равньй индексу однородности самого вложения. Отсюда следует, что все однородные компоненты ненулевого градуированного подмодуля сжимаемого градуированного модуля нетривиальны. Отсюда, в свою очередь, следует, что любой циклический подмодуль сжимаемого градуированного модуля нетривиален. Действительно, пусть $m_{\mu} R=0$ для некоторого $m_{\mu} \in M_{\mu}$, тогда подмодул $m_{\mu} Z+m_{\mu} R$ имеет ровно один ненулевой однородный компонент с индексом однородности $\mu$.

ПРеДЛОЖенИЕ. Для сжимаемого градуированного модуля $M_{R}$ следующие условия әквивалентны: (1) $M_{R}$ - критически сжимаемый; (2) каждый однородный частичный әндоморфизм в $M_{R}$ является мономорфизмом.

Рассмотрим три условия на градуированное колцо $R$ :

(i) кольцо $R$ слабо примитивно

(ii) существуют такие точньй градуированный модуль $M_{R}$, его квази-инъективная оболочка $\bar{M}$, градуированное тело $\Delta=\operatorname{End}_{R}(\bar{M})$, что для произвольного $\Delta_{0}$-независимого однородного набора $v_{1 \phi}, \ldots, v_{k \phi} \in \bar{M}_{\phi}$ найдется такой $0 \neq a_{\alpha} \in \Delta_{\alpha}$, что для произвольного однородного набора $n_{1 \nu}, \ldots, n_{k \nu} \in M_{\nu}$ существует такой $r_{\alpha+\nu+\phi} \in R_{\alpha+\nu+\phi}$, что $a_{\alpha} n_{i \nu}=v_{i \phi} r_{\alpha+\nu+\phi} \in M_{\alpha+\nu}, i=1, \ldots, k$

(iii) существуют такие точньй градуированньй модуль $M_{R}$, его квази-инъективная оболочка $\bar{M}$, градуированное тело $\Delta=\operatorname{End}_{R}(\bar{M})$, что для произвольного $f_{\tau} \in \operatorname{End}_{\Delta}(\bar{M})$ и произволшного однородного $\Delta_{0}$-независимого набора $m_{1 \mu}, \ldots, m_{k \mu} \in M$ найдутся такие $r_{\rho}, s_{\tau+\rho} \in R$, что $m_{i \mu} f_{\tau} r_{\rho}=m_{i \mu} s_{\tau+\rho}$ и $0 \neq m_{i \mu} r_{\rho} \in \Delta_{\rho} m_{i \mu} \forall i$.

Условие (iii) оказьвается слабее условий (i) и (ii), что иллюстрирует простой пример, в котором градуированньй модул удовлетворяет условию (iii), но не удовлетворяет условиям (i) и (ii).

ПримеР. Рассмотрим двумерньй модуль над $\mathbb{Z}_{2}$ как левый модуль над верхнетреугольными матрицами с $\mathbb{Z}_{2}$-градуировкой:

$$
R_{0}=\left\{\left(\begin{array}{cc}
* & 0 \\
0 & *
\end{array}\right)\right\}, \quad R_{1}=\left\{\left(\begin{array}{cc}
0 & * \\
0 & 0
\end{array}\right)\right\} ; \quad M_{0}=\left\{\left(\begin{array}{c}
* \\
0
\end{array}\right)\right\}, \quad M_{1}=\left\{\left(\begin{array}{c}
0 \\
*
\end{array}\right)\right\} .
$$


Условия (i)-(iii) мы назьваем сфформулированньми в терминах "ровной" однородности в силу того, что каждый конечный набор элементов градуированного модуля в этих условиях принадлежит одному компоненту однородности модуля. В работе [2] эти же элементы, являясь также однородными, могут принадлежать разным компонентам однородности. В этом случае условия получаются эквивалентньми.

Дополнительные условия:

(а) существует такой $0 \neq m_{\mu} \in M_{\mu}$, что для каждого индекса однородности (элемента групппы) $\nu$ существуют $\widetilde{m}_{\nu} \in M_{\nu}$ и $\widetilde{r}_{\mu-\nu} \in R_{\mu-\nu}$ такие, что $\widetilde{m}_{\nu} r_{\mu-\nu}=m_{\mu}$, а также $\widehat{r}_{\nu} \in R_{\nu}$ такой, что $m_{\mu} \widehat{r}_{\nu} \neq 0$

(b) все однородные компоненты любого градуированного подмодуля градуированного модуля $M_{R}$ нетривиалшны;

(с) все однородные компоненты любого градуированного циклического подмодуля градуированного модуля $M_{R}$ нетривиальны.

Итак, мы можем сформулировать основной результат.

Расширенная теорема плотности для градуированных по группе колец. Условия (i), (ii), (iii) +(b) и (iii)+(c) на градуированное по группе кольцо $R$ әквивалентны.

Расширенная теорема плотности для градуированных по конечной группе колец. Условия (i), (ii), (iii)+(a), (iii) +(b) u (iii)+(c) на градуированное по конечной группе кольцо $R$ әквивалентны.

Требование к конечности группы $G$ для выполнения условия (а) мотивируется следующим примером, в котором градуированный по $\mathbb{Z}$ модуль $M_{R}$, являясь точным критически сжимаемьг, не удовлетворяет условию (a).

ПримеР. Рассмотрим счетномерньй свободный $\mathbb{Z}$-модул. Выберем счетный базис $\left\{e_{i}\right\}$, где $i$ пробегает все натуральные значения. Обозначим через $M$ подмодуль, натянутьй на базис $\left\{i \cdot e_{i}\right\}$, а через $R$ - подкольцо линейных преобразований исходного $\mathbb{Z}$-модуля, относительно которых подмодуль $M$ инвариантен. Тогда $M_{R}$ - точньй критически сжимаемшй модуль, $M$ и $R$ можно наделить естественной $\mathbb{Z}$-градуировкой. Покажем сжимаемость. Пусть подмодуль $N$ содержит элемент вида $n \cdot e_{k}$, где $n$ и $k$ натуральные. Существует элемент $r$ кольц $R$ такой, что $\left(n \cdot e_{k}\right) r=n \cdot e_{1}$. Следовательно, подмодуль $N_{R}$ содержит циклический $R$-подмодуль $\left(n \cdot e_{1}\right) R$, которьй по сути есть $n \cdot M$, т.е. $\mathbb{Z}$-подмодуль модуля $M$, натянутьй на базис $\left\{n \cdot e_{i}\right\}$, где $i$ пробегает все натуральные значения. Этот циклический $R$-подмодуль очевидным образом изоморфен $M_{R}$. Покажем теперь критическую сжимаемость. Для этого воспользуемся предложением и докажем, что каждьй однородньй частичньй эндоморфизм $f_{\phi}$ в $M_{R}$ является мономорфизмом. Действительно, пусть $f_{\phi}\left(n \cdot e_{k}\right)=0$ для некоторых $n, k \in \mathbb{N}$, тогда $f_{\phi}\left(n k \cdot e_{l}\right)=0$ для любого натуралшного $l$, на котором он определен, а следовательно, $f_{\phi}\left(\mathbb{Z} l \cdot e_{l}\right)=0$, поэтому $f_{\phi}=0$.

Пользуясь случаем, хочу выразить свою благодарность А. В. Михалёву за помощь и внимание.

\section{СПИСОК ЛИТЕРАТУРЫ}

[1] J. Zelmanowitz // Comm. Algebra. 1981. V. 9. № 1. Р. 23-45. [2] С. В. Зеленов // УМH. 2001. T. 56. № 3. C. 167-168.

Московский государственный университет им. М. В. Ломоносова E-mail: sergei@limarenko.mccme.ru 